\title{
The role of mathematics in the learning of physics
}

\begin{abstract}
Physics is the understanding of the laws of nature. Knowledge of these laws not only enables an understanding of the world around us, but is increasingly improving our interaction with it. For instance, technology is the applied use of this knowledge to facilitate communication (GPS, internet), improve quality of life (intelligent TV), and even save lives (MRI). Surprisingly, many people hold fallacious views on the laws of physics, even for simple effects on common, everyday objects. At the other end of the spectrum are experts in physics who perceive gravity as the consequence of curvature of spacetime. The present paper reviews the literature on physics cognition, taking into account key features distinguishing novices from experts to illustrate, theoretically, how education can be effectively geared towards bridging this gap. Of central importance is the fact that physics delves primarily into mathematical concepts. We argue that adequately matching mathematics and physics education supports a parallel evolution of mathematical understanding and its applications in physics; crucial to the development of young scientists and future citizens.
\end{abstract}

Volume 2 Issue I - 2019

\section{Philippe Chassy, Jessica Jones}

Department of Psychology, Liverpool Hope University, UK

Correspondence: Philippe Chassy, Department of Psychology, Mathematical Cognition Research Group, Liverpool Hope University, Liverpool, UK, Email chassyp@hope.ac.uk

Received: October 02, 2018 | Published: January 08, 2019

\section{Naive physics}

Despite the fact that people observe and interact with countless types of objects on a daily basis, assumptions about the effects of forces - or lack of forces - on objects are surprisingly inaccurate, as many studies illustrate erroneous beliefs that people commonly hold. ${ }^{1-}$ ${ }^{3}$ Such misconceptions have been aptly referred to in the literature as naïve physics. ${ }^{4}$ McCloskey et al., ${ }^{1}$ highlighted wrong beliefs about the laws of nature through a study which tasked participants with estimating the trajectory of a metal ball when shot through a curved tube, and the path in which a spinning ball should fall to the ground. Results revealed that nearly half of people with no formal education in physics wrongly assumed the shot ball would follow a curved path, and a third of the participants guessed incorrectly that the falling ball would follow a curved path to the ground. Their results led the authors to suggest that rather than just a mere lack of knowledge, people actually have erroneous preconceptions about motion, developing and applying naïve laws to the behaviors of objects. McCloskey et al., ${ }^{2}$ further exposed misconceptions on motion, such as the commonlyheld belief that an object - if dropped by a moving carrier - will fall to the ground in a straight line. Although the laws of motion entail that such an object should fall to the ground in an arc, over half of participants believed that the ball would either fall in a straight line $(49 \%)$ or backwards $(6 \%)$. In another field of classical mechanics, naïve notions have also been documented in ideas about speed. ${ }^{3,5}$ More recently, naïve beliefs about reflection have been exposed. ${ }^{6,7}$ Due to the centrality of physics principles in the design of cars, aeroplanes, medical devices, and many other crucial aspects of daily life, it is of some concern that many beliefs regarding the order of the world in which we live are wrong.

\section{Physics expertise}

Though it is well documented that people hold incorrect beliefs about the basic principles of mechanics, other research has focused on the opposite end of the spectrum: Physics expertise. In a seminal experiment, Chi et al., ${ }^{8}$ investigated physics knowledge, by comparing the problem-solving abilities of advanced $\mathrm{PhD}$ physics students with novice undergraduates in a series of tasks. While it was found that novices categorize and approach problems based on literal, surface features, experts categorize and approach problems based on underlying, abstract, physics principles. Such evidence supported the idea that novices and experts use different levels of conceptualization when building a representation. Not only do experts and novices in physics attend to different aspects of a problem, i.e. surface features vs. abstract principles, they also employ different approaches in problem-solving; as where the novice typically works backwards, the expert often works forwards. ${ }^{9}$ A key finding of Chi et al.'s research is that knowledge has a profound influence on the ways in which an expert approaches a problem within their domain of expertise, where activated knowledge is used to process current information. Theories of expert intuition which consider both the behavioral findings of expertise development in addition to biological mechanisms, highlight the central role of conceptual knowledge in quick problem-solving within a broad range of fields of expertise. ${ }^{10}$ Intuition in a domain is achieved when an individual is able to 'see' the key features of a situation, according to which a solution is rapidly retrieved. Rapid recognition and accurate problem-solution is possible only when the expert has developed highly sophisticated concepts, rich in both abstract and concrete knowledge. Thus, intuitive problem-solving in a domain requires accumulation of a wealth of knowledge, and as such increases as a function of expertise. In physics, most problems are both posed and solved with mathematics. Hence, in order to reach fluid problem-solving in physics, one must come to master the necessary mathematical concepts describing the principle.

\section{Mathematics in physics}

In order to consider the progression from a naïve, novice level in physics to the deep understanding displayed by the expert, it is necessary to examine the type of knowledge needed to adequately process abstract physics information. A deep, integrated understanding of concepts in physics cannot exist without understanding their mathematical descriptions. Let us consider, for example, the simple laws of classical mechanics. The physics of a point is generally described by a series of equations. The simplest one, usually introduced early in the curriculum, is $\mathrm{d}=\mathrm{vt}$, where $d$ is the distance travelled in meters, $v$ the speed in meters per second, and $t$ is the time in seconds. Students are typically asked to find either $d$ or $v$, and are provided with the other quantities. Thus, at the core of mastery of this simple 
equation lies a solid understanding of multiplication and division. This concurrent mathematical understanding becomes more advanced in line with the increasing complexity of physics concepts. For example, to improve their understanding of classical mechanics further, pupils and students need to learn more sophisticated mathematical concepts like the derivative and the integration. Only then can they be introduced to the notion of acceleration and the relationship between acceleration, speed, and change of position. To take an example, from the simplest formula of a falling object (air resistance being neglected), $\mathrm{y}=-1 / 2 \mathrm{gt}^{2}$, with $\mathrm{g}=9.81 \mathrm{~m} \mathrm{~s}^{-2}$, students can learn position change as a function of the $\mathrm{g}$ force on Earth. From this, they can play with derivatives in order to construct an internal representation of how speed varies as a function of time. The above highlights the central fact that the accuracy of the mental representation of the law of motion is embedded into the mathematical concepts describing it. Hence, understanding the mathematical concept is central to understanding the physics phenomenon. This simple example illustrates the fact that physics, in describing reality, uses mathematical tools which, in turn, offer an accurate description of reality. This point holds true for all physics principles. The theory of electromagnetism is dominated by the Maxwell equations, Einstein's equations describe the law of the universe at the highest scale, and quantum mechanics describes the behaviour of particles at the smallest end of the scale. In short, the tight link between mathematics and physics can be found at every corner.

\section{Expertise acquisition}

A key question concerning education in physics is how long it actually takes to gain a good understanding of physics principles. A sound, immediate understanding of physics is reached when the main features of a difficult problem are identified and a solution is retrieved within seconds. ${ }^{10}$ As mentioned, intuition in physics is made increasingly possible as a function of expertise. For those who reach expert levels of performance, the bulk of the research indicates that amongst all of the factors, it is training that plays the major role in performance. ${ }^{11-13}$ The estimates are that, to reach expertise level, one should dedicate around 10,000 hours to a given activity Ericsson et al., ${ }^{12}$ to acquire around 300,000 chunks of knowledge. ${ }^{11}$ This figure may seem audacious at first sight. However, spread over several years, such an accumulation of hours is in fact attainable. For instance, consider a child enters primary school at 6 , goes onto university at around 18, and later completes a $\mathrm{PhD}$ (and hence reaches the highest level of academic expertise) by the age of 26 . With these figures in mind, we can compute the number of days from the onset of primary school education to completing the $\mathrm{PhD}$ to be 7,300 days $(=20 * 365$, neglecting leap years). Thus, over the 7,300 days of study, to achieve 10,000 hours' worth of practice in physics (or any given domain), one must dedicate approximately 1 hour 24 minutes per day; around 10 hours per week. This too, appears to involve a huge amount of work. However, it is worth bearing in mind that these estimations pertain to the highest levels of expertise. Likely, more common, practical knowledge of physics will require less amounts of work. Irrespective of the exact number of hours, the real concern within a given education system should be how to best utilize the time dedicated to mathematics and physics; whilst keeping in mind that performance is a function of training, and thus more practice should increase performance.

\section{Mathematical learning}

Since mathematics is central to physics expertise, it is important to consider how mathematical skills are acquired. Research on infants
Starkey et al., ${ }^{14}$ babies Antell et al., ${ }^{15}$ and even animals strongly suggests that both animals and humans naturally possess the ability to distinguish quantities. ${ }^{16,17}$ Our basic form of number representation arises from the Approximate Number System (ANS). ${ }^{18}$ The ANS enables discrimination between sets of quantities; for example, it is the system by which it seems obvious that ten items are more than five. The system is not very accurate if it does not receive proper training. Extensive evidence shows that it takes several years of practice to actually master the natural numbers and that this ability is usually predictive of later academic success in primary school level mathematics. The ability to discriminate quantities has been shown to evolve with age, ${ }^{19}$ indicating that the accuracy of the ANS sharpens throughout development. In neurotypical developing children, the ANS reaches high levels of acuity during early adolescence. ${ }^{19}$ Although studies confirm that precision occurs organically - as shown across cultures with differences in education - ANS acuity can be significantly improved through practice and education..$^{20,21}$ The notion of natural number, though initially supported by a hardwired system, still requires populations of neurons to fine-tune their activity through learning so as to respond to specific quantities. This system, expressing concrete quantities, will then be used to construct more complex mathematical knowledge, ultimately expressed in symbolic format. The ever increasing complexity of the concepts increases the volume and level of abstractedness of the semantic network holding these concepts. Ultimately, the database of concepts can be used to account for a wide variety of phenomena in physics.

\section{Education in physics and mathematics}

The important implication from the above is that the notion of number - being the basis upon which all mathematical cognition rests - is the crucial component in mathematical performance. From this, it is feasible that an optimum strategy in mathematical educational policy may be that, instead of overloading students with countless concepts, teaching should begin slowly to ensure that the basic representation of numbers and quantities has been appropriately acquired before progressing onto more complex ideas. It is upon such a solid base that further, more elaborated concepts, should rest. For example, it has recently been shown that the notion of negativity, used to represent abstract mathematical quantities such as negative numbers, also recruits the region of the brain known to process natural numbers. ${ }^{22}$ If the foundation of mathematical knowledge is not robust, the store of abstract mathematical concepts will not enable correct understanding of more complex, mathematical problems. If teaching is adapted to the individual differences of students at the earliest age, it should be possible to equip more people with a strong basis of mathematics. Thus, encouraging a strong understanding of the basics should engender more positive ideas towards mathematics, increasing understanding and positively influencing learning within a population. A flexible approach to maths in the early stages of learning should reduce the number of pupils who disengage.

In line with this approach, physics education should also aim to first cement a basic understanding, before exposure to more advanced aspects. Following this, physics could be divided into its conceptual and mathematical components. Though both are entwined it is possible to separate them for pedagogic purposes. Specifically, it would be advisable to initially present the conceptual aspect; allowing the mind of the pupil to mature before the mathematics are presented. Introducing concepts in physics early on in the curriculum, in parallel with basic mathematics, should prevent the development of false conceptions. As soon as possible thereafter, once conceptual aspects 
of physics problems and a basic understanding of math are likely achieved, it is advisable to introduce the mathematical component of a physics problem; allowing students to improve their understanding, through seeing how quantities help to clarify the concepts and enable problem-solving. Although students must first grasp conceptual physics whilst being equipped with a solid basis of foundational mathematics, the younger a pupil the better, since a developing brain has a higher ability to absorb information than an average adult; and so, a good balance of a student understands and their age is key to the successful implementation of the suggested approach. Such an approach should constitute a sound basis for future intuition in physics.

The theoretical and empirical elements reported in the above indicate that mathematics and physics should be deliberately taught in parallel. It is crucial to take into account a pupil's age to establish the kinds of concepts, whether concrete or abstract, that they are able to grasp. The education system should align the physics and mathematics curricula in such a way that pupils can immediately make sense of the mathematical concepts and their applications. ${ }^{23}$

\section{Acknowledgments}

None.

\section{Conflicts of interest}

The authors declare that there is no conflict of interest.

\section{References}

1. Mc Closkey M, Caramazza A, Green B. Curvilinear motion in the absence of external forces: Naive beliefs about the motion of objects. Science. 1980;210(4474):1139-1141.

2. Mc Closkey M, Washburn A, Felch, L Intuitive physics: The straightdown belief and its origin. Journal of Experimental Psychology: Learning, Memory, and Cognition. 1983;9(4):636-649.

3. Rohrer D, Misconceptions about incline speed for nonlinear slopes Journal of Experimental Psychology: Human Perception and Performance. 2002;28(4):963-973.

4. Mc Closkey M, Kohl D. Naive physics: the curvilinear impetus principle and its role in interactions with moving objects. Journal of Experimental Psychology: Learning, Memory, and Cognition. 1983;9(1):146-156.

5. Rohrer D, The natural appearance of unnatural incline speed. Memory \& cognition, 2003;31(5):816-826.

6. Bertamini M, Parks TE. On what people know about images on mirrors. Cognition. 2005;98(1):85-104.
7. Lawson R. People cannot locate the projection of an object on the surface of a mirror. Cognition. 2010;115(2):336-342.

8. Chi MT H, Feltovich PJ, Glaser R. Categorization and representation of physics problems by experts and novices. Cognitive Science. 1981;5(2):121-152.

9. Larkin J, Mc Dermott J, Simon DP, Simon HA. Expert and novice performance in solving physics problems. Science. 1980;208(4450):13351342.

10. Chassy P,Gobet F. A hypothesis about the biological basis of expert intuition. Review of General Psychology. 2011;15(3):198.

11. Bilalic M, Mc Leod P, Gobet F. Does chess need intelligence?-A study with young chess players. Intelligence. 2007;35(5):457-470.

12. Ericsson E A, Krampe RT, Tesch Romer C. The role of deliberate practice in the acquisition of expert performance. Psychological review. 1993;100(3):363-406.

13. Grabner RH, Stern E, Neubauer AC. Individual differences in chess expertise: A psychometric investigation. Acta psychologica, 2007; 124(3):398-420.

14. Starkey P, Cooper R G. Perception of numbers by human infants. Science. 1980;210(4473):1033-1035.

15. Antell SE, Keating DP. Perception of numerical invariance in neonates. Child development.1983;54(3):695-710.

16. Brannon EM, Terrace HS. Ordering of the numerosities 1 to 9 by monkeys. Science. 1998;282(5389):746-749.

17. Uller C, Jaeger R, Guidry G, et al. Salamanders (Plethodon cinereus) go for more: rudiments of number in an amphibian. Animal cognition. 2003;6(2):105-112.

18. Piazza M. Neurocognitive start-up tools for symbolic number representations. Trends in cognitive sciences. 2010;14(12):542-551.

19. Halberda J, Feigenson L. Developmental change in the acuity of the "Number Sense ": the Approximate Number System in 3-, 4-, 5-, and 6-year-olds and adults. Developmental psychology. 2008;44(5):14571465 .

20. Piazza M, Izard V, Pinel P, Le Bihan D, et al. Tuning curves for approximate numerosity in the human intraparietal sulcus. Neuron. 2004;44(3):547-555.

21. Piazza M, Pica P, Izard V, et al. Education enhances the acuity of the nonverbal approximate number system. Psychological Science. 2013;24(6): 1037-1043.

22. Chassy P, Grodd W. Comparison of quantities: core and format-dependent regions as revealed by fMRI. Cerebral Cortex. 2012;22(6):1420-1430.

23. Gobet F, Simon HA. Five seconds or sixty? Presentation time in expert memory. Cognitive Science. 2000;24(4):651-682. 